\title{
SARCOCYSTOSIS IN MEAT-PRODUCING ANIMALS: AN UPDATING ON THE MOLECULAR CHARACTERIZATION
}

\author{
Bushra Hussain Shnawa ${ }^{1,2^{*}}$ and Sara Omer Swar ${ }^{3}$ \\ 'Department of Biology, Faculty of Sciences, Soran University, Kurdistan, Iraq \\ ${ }^{2}$ Scientific Research Center, Soran University, Kurdistan, Iraq \\ ${ }^{3}$ College of Agricultural Engineering Sciences, Salahaddin University, Kurdistan, Iraq \\ *Corresponding author: Bushra.shnawa@soran.edu.iq
}

\section{INTRODUCTION}

Sarcocystosis is a globally distributed zoonotic protozoan disease that infects a wide variety of mammals, reptiles, and birds. This disease is caused by Sarcocystis species, which are a coccidian intracellular protozoan parasite of the genus Sarcocystis that belongs to the family Sarcocystidae of the phylum Apicomplexa (Fayer et al. 2015). About 200 valid species of Sarcocystis have been identified, which vary in their pathogenicity to the host, ranging from avirulent to highly virulent; some species are zoonotic; however, complete life cycles are known only for 26 species (Dubey 2015; Dubey et al. 2016).

This parasite has an obligatory two-host life cycle (prey and predator) known as intermediate and definite hosts. The asexual part of its life cycle is completed in the intermediate host, which is usually a herbivore animal. At the same time, sexual stages of its development occur in the definite or final host, a carnivore or omnivore animal (Lindsay and Dubey 2020). Sarcocystis is non-pathogenic in the definite host, and several species are also nonpathogenic in the intermediate hosts. In general, species transmitted by the canid's definite host are pathogenic, while those transmitted by the felid's definite host are non-pathogenic (Lindsay and Dubey 2020).

Human infection with Sarcocystis spp. is relatively rare. Still, humans serve as definite or intermediate hosts for this parasite. They become the final host by consuming under-cooked beef or pork meat, infected with $S$. homonis and S. suihominis, respectively. Symptoms of human intestinal Sarcocystis infection include nausea, abdominal discomfort, stomach ache, and diarrhea (Fayer et al. 2015). Humans can become an intermediate or aberrant host for seven or more Sarcocystis species, including S. nesbitti, which was identified in humans through18SrDNA sequence analysis. $S$. nesbitti infection is considered as a new zoonotic disease caused by the ingestion of food or water contaminated with this Sarcocystis species. Moreover, S. heydorni, which mostly infects cattle, is also considered as a zoonotic protozoan (Dubey 2015; Dubey et al. 2016). Infection with Sarcocystis protozoan in the intermediate host can lead to morbidity, mortality, abortions, decreased meat production, and increased meat condemnation due to presence of macrosarcocysts. Up to now, the importance of human sarcocystosis has failed to gain necessary attention regardless of the potential for muscular or intestinal Sarcocystis infection to infect a considerable number of people (Fayer 2004; Tappe et al. 2013).

Sarcocysts caused by sarcocystosis are common in many domestic and wild animals and can cause mortality in these infected animals. There are two types of Sarcocystis cysts, the microscopic and the macroscopic sarcocysts (Dubey 2015).

Sarcocystosis is also considered to be one of the most prevalent intracellular protozoan diseases of livestock (Fayer 2004). Animals including cattle, goats, and sheep are susceptible to sarcocystosis (Dubey and Lindsay 2006) and are considered as intermediate hosts. Cysts are only formed within the muscles of the intermediate hosts. Therefore, they are called sarcocysts, which commonly indicate a wide-ranging hosts and worldwide distribution (Abdel-Ghaffar et al., 2009). Several species of Sarcocystis develop macroscopic sarcocysts in the tissue of domesticated animals. Amongthese, S. caprafelis (syn. S. moulei) is found in goats, while $S$. gigantea (syn. $S$. ovifelis) and $S$. medusiformis in sheep (Dubey and Lindsay 2006).Similarly, $S$. hirsuta and $S$. hominis form macroscopic sarcocysts in cattle, while $S$. buffalonis and $S$. fusiformis form these cysts in the water buffalo (Lindsay and Dubey 2020).

\section{History of Sarcocystosis}

In 1843, milky white threads in the muscles of a deer mouse, Mus muscula, were observed by Friedrich Miescher in Switzerland (Miescher 1843). These threads were known as Miescher's tubules for many years. Later, the causative organism of the problem was named as Sarcocystis after Lankester (1882), a Greek word derived from "sarkos" that means muscle or flesh, and "kystis" meaning bladder, describing the parasite's form that encysted in the tissue of the intermediate hosts (Fayer 2004). Thereafter, S. muris was the first species named in the genus Sarcocystis by the Swiss scientist Friedrich Miescher. The house mouse was the only known intermediate host for this organism (Ruiz and Frenkel 1976). In 1967, crescent-shaped structures typically found in cultures of sarcocystiswere investigated and the organism was identified as a protozoan, a close relative of Toxoplasma spp. and Eimeria spp. (Fayer 2004).

In 1969, Mandour identified a new Sarcocystis species in rhesus macaques named $S$. nesbitti, after Mr. Nesbitt, who 
observed the trophozoites in stained smears. The definite hosts of $S$. nesbitti are now-a-days known to be snakes, whereas several primates, including human beings, can be intermediate hosts (Mandour 1969).

Scientists debated whether the Sarcocystis spp. were protozoa until 1967, after the first Sarcocystis report when the spindle or crescent-shaped bodies (bradyzoites) in the sarcocystis were studied by electron microscopy. Its organelles resembled to other apicomplexan protozoa, such as Toxoplasma and Eimeria (Fayer 2004).

The terminology for Sarcocystis spp. was suggested by combining the Latin names of both intermediate and definite hosts, such as Sarcocystis ovicanis (S. tenella), for which sheep is an intermediate host, while the dog acts as a final host.Similarly, S. ovifelis (S. gigantea) in which sheep serves as an intermediate host, while the cat is the final host. S. bovicanis comes from two Latin words: bos for cattle and canis for dog (Mehlhorn 2016). Some historical landmarks concerning Sarcocystis are listed in Table 1.

\section{Classification of Sarcocystis species}

According to the classification system proposed by Levine (1986), Sarcocystis parasite is classified as:

$\begin{array}{ll}\text { Phylum Apicomplexa } & \text { Levine (1970) } \\ \text { Class Sporozoasida } & \text { Leuckart (1879) } \\ \text { Subclass Coccidiasina } & \text { Leuckart (1879) } \\ \text { Order Eucoccidiorida } & \text { Leger and Duboscq (1910) } \\ \text { Suborder Eimeriorina } & \text { Leger (1911) } \\ \text { Family Sarcocystidae } & \text { Poche (1913) } \\ \text { Subfamily Sarcocystinae } & \text { Poche (1913) } \\ \text { Genus Sarcocystis } & \text { Lankester (1882) }\end{array}$

Life cycle of Sarcocystis species

The life cycle of Sarcocystis species was unknown until 1972, when some investigators ultrastructurally studied the gametogonic and oocyst formation properties of $S$. falctula in poultry during in vitro experiments (Fayer 1972; Rommel et al. 1972). In 1973, Wallance (1973) fed experimental mice with coccidia collected from the feces of a naturally infected cat and thus, he induced sarcocysts formation in mice.

The life cycle of Sarcocystis species needs two obligatory prey-predator hosts for its completion, intermediate host and definite host, followed by one another sequentially and designated as a di- heterogeneous parasite (Odening 1998) (Fig. 1). The stages occurring during the life cycle of Sarcocystis species are as follows:

\section{Asexual stages}

The asexual stages of Sacocystis parasite development occur only in the intermediate host, which is generally a prey animal. The infection starts when these animals ingest Sacocystis oocysts or sporocysts from the food or water contaminated with feces of the final host. Sporozoites are released from the ingested sporocysts by the action of trypsin and bile. The free sporozoites invade the gut wall and lodge themselvesin the endothelial cells of the small arteries. Four cycles of asexual reproduction are distinguished. Several nuclear divisions occur in the sporozoites, followed by segmentation to generate merozoites, which are motile and crescent-shaped. Following these schizonts' cycles, the Sarcocystis encysts itself in the muscles and form metrocytes, which are changed to bradyzoites. Sarcocyst having bradyzoites indicates the last encysted stage in the skeletal, cardiac, and smooth muscles of infected herbivores, infectious for carnivorous animals as definite hosts (Dubey 2015; Fayer et al. 2015; Dubey et al. 2016).

Two types of tissue sarcocysts can occur in sheep, goats, and cattle; either as microscopically or macroscopically visible structures of different Sarcocystis spp, as shown in Fig. 2 and Fig. 3. Fig. 4 illustrates the Banana-shaped bradyzoites prepared by muscle mincing and squash methods of fresh esophageal tissue from infected sheep and goats and stained with Giemsa stain.

\section{Sexual stages}

A definite host acquires the infection by ingesting mature sarcocysts from muscles of an infected animal (Lindsay and Dubey 2020). These sarcocysts are digested in the digestive system of the definite host and release the bradyzoites, which invade mucosa of the small intestine. Then, they are transformed into male and female gametes, called microgametes and macrogametes, respectively. After fertilization, these gametes form a zygote, which develops into the non-motile oocysts. The oocysts sporulate in the small intestine. The sporulated oocysts are thin-walled, each having two sporocysts: Each sporocyst contains four sporozoites. Then, it ruptures, liberating the sporocysts into the lumen of intestine; these sporocysts are defecated with feces (Lindsay and Dubey 2020), as has been shown in Fig. 1.

\section{Ultrastructure of Sarcocystis spp}

Sarcocystis spp. are single-cell eukaryotic organisms that contain a nucleus, nucleolus, endoplasmic reticulum, ribosomes, Golgi apparatus, and mitochondria. They also have the characteristic apicomplexan organelles, such as the apical rings (conoidal rings), polar ring, a conoid, pellicle, subpellicular microtubules, micropores, rhoptries, and micronomes, as shown in Fig. 5 (Ghaffar et al. 1989; Al-Quraishy et al. 2014; Dubey et al. 2016).

The intermediate host becomes infected by ingesting the sporocysts, the latter release sporozoites in the small intestine. These sporozoites appear in the mesenteric arteries and lymph nodes, where they eventually liberate merozoites into the blood, initiating the development of the sarcocysts in muscle. Resultantly, bradyzoites areformed, which is known as the infective stage for the consumer (Markus et al. 2004).

A sarcocyst plays a significant role in the transmission, as well as in the taxonomy, of Sarcocystis spp. It results from the invasion of merozoite into a myocyte or neural cell. Then the merozoite becomes rounded to change into a 
metrocyte, with several organelles of the apical complex, like the micronemes, conoid and apical rings disappear. In contrast, ribosomes, endoplasmic reticulum and mitochondria become more abundant, and the nucleus becomes larger (Dubey et al. 2016). Within the cysts, the parasite multiplies by endodyogony or endopolygony, leading to the formation of metrocytes and later thousands of infectious cystozoites (bradyzoites), as shown in Fig. 6.

\section{Sarcocystis species that are infecting ruminants}

Sheep, goats and cattle are ungulates, 'hooved' animals that are members of the Order Artiodactyla (animals with cloven hooves), suborder Ruminantia (ruminants or cudchewing animals), and Family Bovidae. These animals are herbivores, and they meet all their glucose requirements from gluconeogenesis. The subfamily Capra includes sheep and goats (Underwood et al. 2015).

Numerous spp. of Sarcocystis infect sheep, some of them are transmitted via canids and others by feline (cat). The species transmitted by dogs are mostly pathogenic and produce microsarcocyst in the skeletal and cardiac

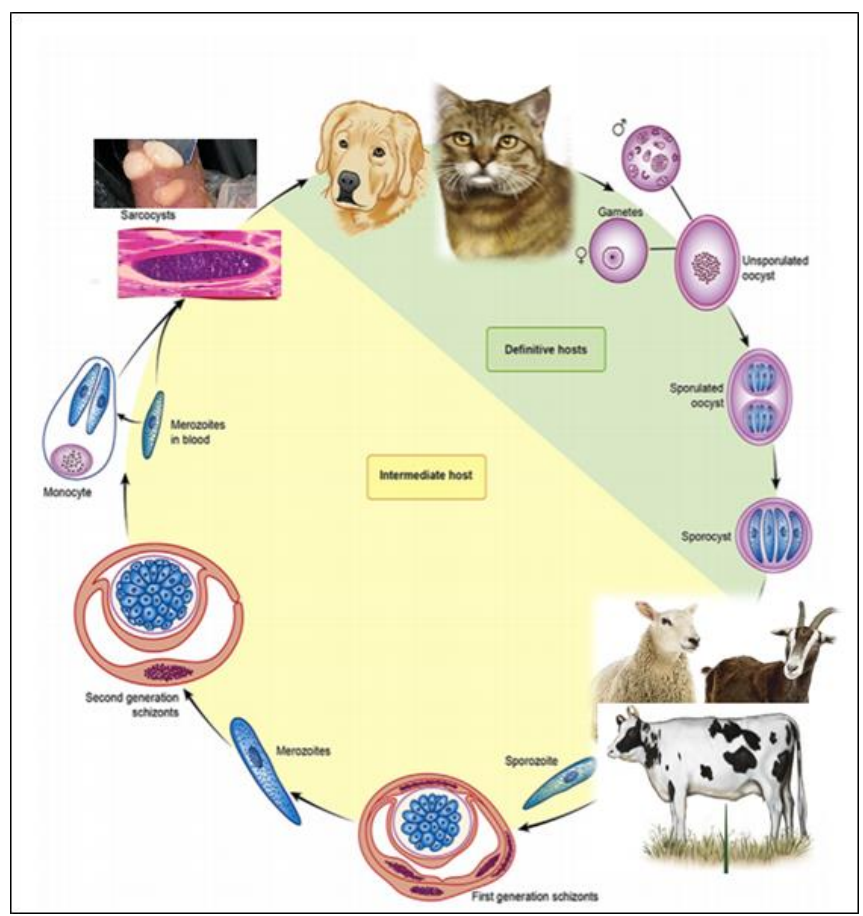

Fig. 1: The life cycle of Sarcocystis spp. from ruminants (Modified from Lindsay and Dubey 2020; Swar and Shnawa 2021).

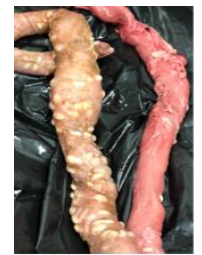

A

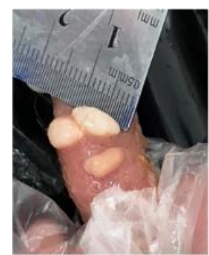

B

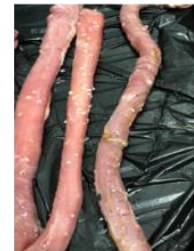

C

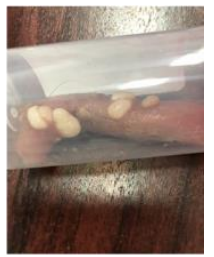

D
Fig. 2: Appearance of macroscopic sarcocysts in the esophagi of goats (A\&B) and sheep (C\&D) (Swar and Shnawa 2021). Note several cysts appear with different sizes and shapes on the surface of infected esophagus. muscles of the animal. These species, like $S$. tenella, can lead to pathological effects in sheep, like anorexia, anemia, weight loss, abortion, neural symptoms, and even death (Dubey et al. 1988; Abdel-Baki et al. 2009). However, the species transmitted by cats, for instance $S$. gigantea and $S$. medusiformis, form macrosarcocyst in the esophagus, tongue, and larynx; the pathological effects of macrosarcocyst are more severe than those of the microsarcocysts (Collins et al. 1979; Dubey et al. 2016).
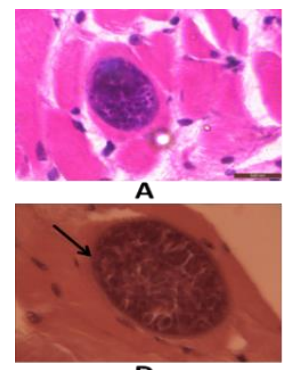

D

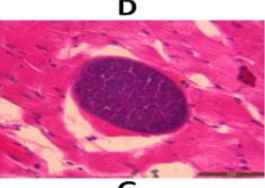

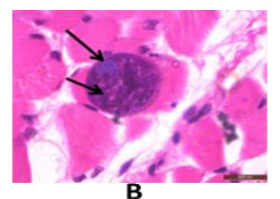
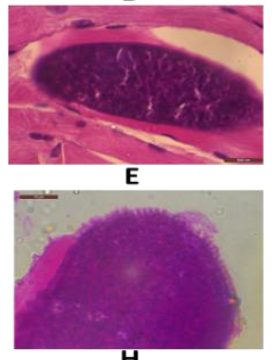
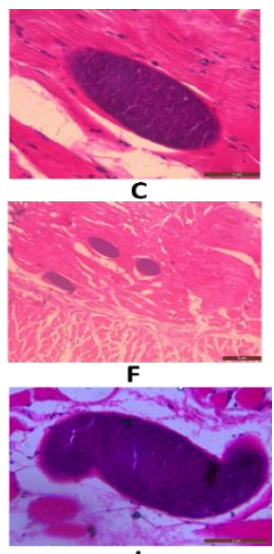

Fig. 3: Microscopic sarcocysts within the esophagi of infected sheep and goats (Swar 2021).

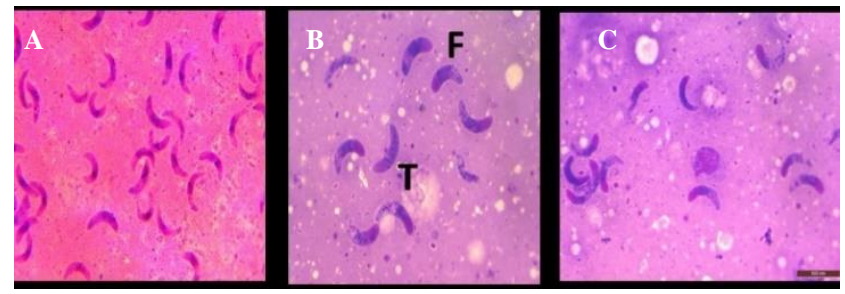

Fig. 4: Banana-shaped bradyzoites by muscle mincing and squash technique of fresh esophageal tissue from sheep and goats stained with Giemsa stain (A, B, and C). Bradyzoites from macroscopic sarcocyst of goats and sheep. B also shows fat (F) and thin (T) types of bradyzoites. Scale bar =50onm. (Swar 2021).

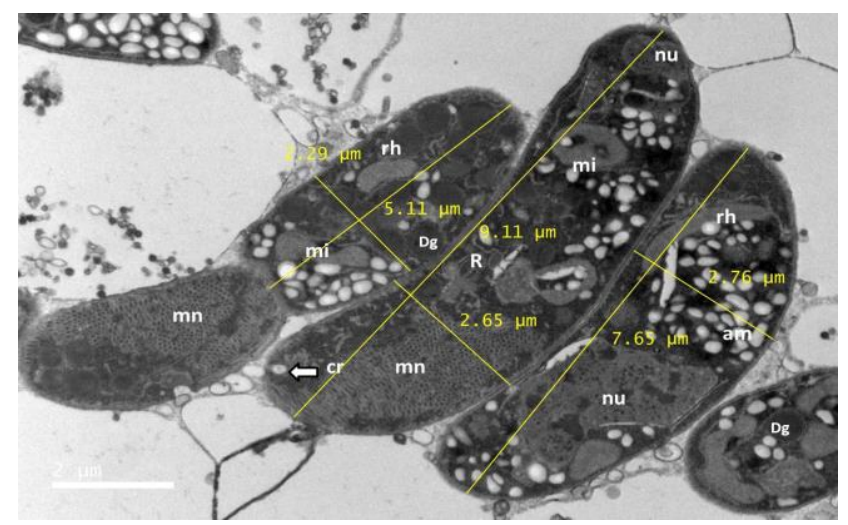

Fig. 5: Transmission electron micrograph of a longitudinal section of macroscopic sarcocyst of Sarcocystis spp. of sheep shows bradyzoites. Note conoidal ring (cr), amylopectin (am), numerous micronemes $(\mathrm{mn})$, subterminal nucleus $(\mathrm{nu})$, rhoptries (rh), dense granules as black structure (white arrow), amylopectin as white granules (am), ribosome(R) and mitochondrion (mi). Scale bar $=2 \mu \mathrm{m}$. (Swar and Shnawa 2020). 
Three Sarcocystis species have been identified in domestic goats; these include $S$. caprafelis (synonym $S$. moulei), S. hiricanis, and $S$. capracanis. The $S$. hiricanis and $S$. capracanis are usually associated with microscopic sarcocysts, whereas $S$. moulei produces macroscopic cysts (Dubey et al. 2016). Medically, $S$. capracanis shows more severe pathological effects than those of the other two species (Collins and Charleston 1979). The infected goats may show fever, anorexia, weight loss, tremors, abortion, and death in severe cases (Dubey et al. 1981). However, some investigations documented the infection of sheep and goats with Sarcocystis species that are unusual in these hosts, such as the infection of sheep with $S$. moulei that commonly infects goats in Saudi Arabia (Al-Hoot et al. 2005) and Iran (Kalantari et al. 2016). Similarly, $S$. gigantean, which usually infects sheep, can also cause infection in goats (Ghaffar et al. 1989) and it was suggested that goats can be a host for three species of Sarcocystis described as $S$. moulei, including $S$. ovifelis ( $S$. gigantea). Moreover, using molecular and ultrastructural techniques, Hong et al. (2016) evidenced the infection of Korean goats with $S$. tenella, which is commonly known as sheep specific.

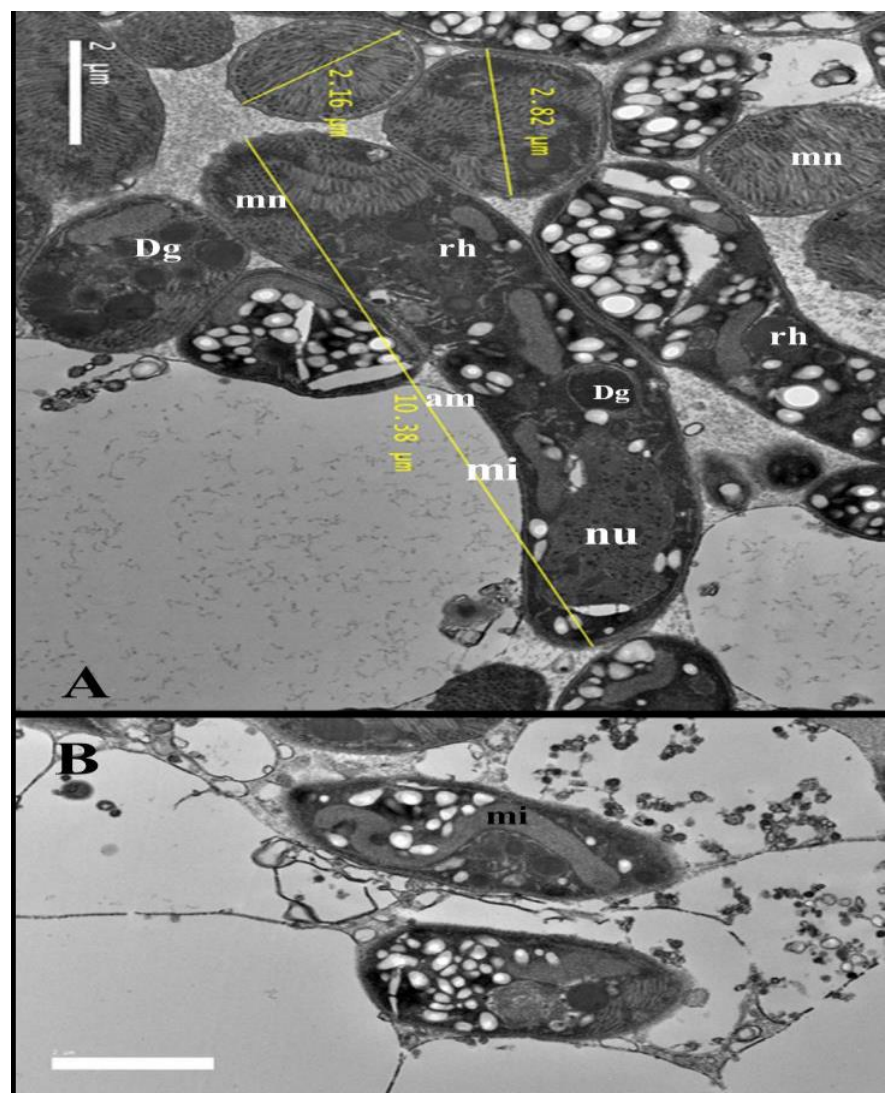

Fig. 6: Transmission electron micrographs of macroscopic sarcocyst of Sarcocystis spp from sheep: (A). Longitudinal and cross-sections of bradyzoites showing conoidal ring (cr), amylopectin (am), micronemes (mn), nucleus (nu), and dense granules (dg), and mitochondrion. Scale bar $=2 \mu \mathrm{m}$. (B). Crosssection of bradyzoite with one long twisted mitochondrion (mi), nucleus (nu), dense granules (dg), and amylopectin as white granules (am). The parasite is surrounding by a membrane of parasitophorous vacuole within the host cell cytoplasm-scale bar $=2 \mu \mathrm{m}$ for both micrographs (Swar 2021).
Five species of Sarcocystis have been identified in cattle: these include Sarcocystis cruzi, Sarcocystis heydorni, Sarcocystis hirsuta, Sarcocystis hominis, and Sarcocystis rommeli; the definite hosts for these species are canines (S. cruzi), felines (S. hirsuta, $S$. rommeli), and primates $(S$. heydorni, S. hominis).

Generally, four Sarcocystis species can infect water buffalo, including $S$. buffalonis, $S$. dubeyi, S. fusiformis, and S. levinei.

\section{Molecular Characterization of Sarcocystis spp}

In recent years, researchers gained outstanding achievements in several molecular procedures to identify various Sarcocystis spp that can infect different animals and are recognized as host-specific. These molecular procedures include $18 \mathrm{~S}$ rRNA, 28S rRNA, 18S rDNA, mitochondrial cytochrome $\mathrm{C}$ oxidase subunit 1 gene $\left(\mathrm{COX}_{1}\right)$ and ITS-1 region (Dubey et al. 2014; Blazejewski et al. 2015; Ng et al. 2015; Hu et al. 2017; El-Morsey et al. 2019). The first genetically identified species of the genus Sarcocystis through genome sequence was the $S$. neurona. Its genome contains $127-\mathrm{Mbp}$, and its size is twice that of the size of other coccidian genomes (Blazejewski et al. 2015). Tenter et al. (1992) recognized two monophyletic groups of Sarcocystis spp. One group represents the species for which cats arethe definite hosts. In contrast, the second group has the species that need dogs as definite hosts in their life cycles.

Previously, Sarcocystis spp. were known to be primarily host-specific, but, during the last few years, a large number of Sarcocystis spp which use different animals as intermediate hosts were identified. Consequently, host specificity for various Sarcocystis spp. is questionable. In this regard, Al-Hoot et al. (2005) characterized ultrastructurally the $S$. moulei in sheep infection, the species which usually infects goats in Saudi Arabia. Moreover, in Iran, S. moulei was documented genetically in sheep (Kalantari et al. 2016). Similarly, S. capracanis was identified from the cerebrospinal fluid of sheep suffering from meningoencephalitis in the United Kingdom (Formisano et al. 2013). Based on these findings it can be suggested that sheep can act as an alternative intermediate host for these species in addition to goats. In a study regarding Sarcocystis infection from Egypt, Elmishmishy et al. (2018) documented the resemblance of $S$. gigantea from sheep with $S$. moulei from goats. They proposed the cross-transmission of $S$. moulei amongst sheep and goats, and suggested them to be phylogenetically related. Other researchers confirmed the genetic similarity between $S$. tenella of sheep and $S$. capracanis from goats and considered them as sister species (El-Morsey et al. 2019).

Moreover, Saudi Arabian researchers also revealed the presence of phylogenetic association among Sarcocystis spp from both hosts, sheep and goats (Metwally et al. 2019). According to Yang et al. (2001), morphologically identical species from two different intermediate hosts should be classified as similar species. However, many Sarcocystis spp. seem to have a more comprehensive intermediate host opportunity than previously recognized. 
Table 1: Historical landmarks regarding Sarcocystis, modified from Dubey et al. (2016)

Scientist's name Year The outcomes

Miescher $\quad 1843$ Sarcocystis reported from the muscle of house mouse

Lankester $\quad 1882$ The genus Sarcocystis named

Fayer $\quad 1972$ Sexual stages cultured in vitro

Rommel et al. 1972 Two obligatory hosts required for completion life-cycle

Fayer \& Johnson 1973 The pathogenicity proved with recognition of vascular phase

Heydorn $\quad 1975$ Several Sarcocystis species are recognized within a given host.

Fayer \& Johnson 1975 Chemotherapy (Amprolium) is documented

Fayer et al.

Dubey et al.

Dubey et al.

Dubey et al.

1976 Abortion results from Sarcocystis infection in cows

1981 Protective immunity against sarcocystosis is proved in goats

1989 Classification of Sarcocystis species depending on cyst wall morphology is recommended.

1991 First detection of the causative agent of equine protozoan myeloencephalitis and classified as Sarcocystis neurona.

Blazejewski et al. 2015 First characterization of Sarcocystis genome that related to S.neurona.

Table 2: Several recent articles concerning the molecular characterization of Sarcocystis spp. of meat-producing animals.

\begin{tabular}{|c|c|c|c|c|}
\hline Sarcocystis spp. & Molecular technique & Host & Country & Reference \\
\hline S. tenella & RFLP-PCR for the $18 \mathrm{~S}$ rRNA & Sheep & Brazil & (da Silva et al. 2009) \\
\hline $\begin{array}{l}\text { S. fusiformis, S.cruzi, } \\
\text { S. homonis, S. hirsuta }\end{array}$ & RFLP-PCR for 18S rDNA & $\begin{array}{l}\text { Cattle \& Water } \\
\text { buffalo }\end{array}$ & Vietnam & (Jehle et al. 2009) \\
\hline S. gigantea and S. tenella. & Mitochondrial cytochrome c & Norwegian & Norway & (Gjerde 2013a) \\
\hline S. hirsuta and $S$. sinensis & oxidase subunit I gene (coxı) & Sheep & & \\
\hline & and the nuclear ssr RNA & Argentinean cattle & & \\
\hline S. tenella & $\begin{array}{l}\text { 18S r RNA gene sequence with } \\
\text { PCR-RFLP }\end{array}$ & Sheep & Iran & (Shahbazi et al. 2013) \\
\hline S. cafferi & 18S Rrna\& COX & Buffalo & \multicolumn{2}{|c|}{ South Africa (Dubey et al. 2014) } \\
\hline S. gigantea and S. tenella & 18S rRNA & Sheep & Iran & (Bahari et al. 2014) \\
\hline S. gigantea and $S$. medusiformis & 18S rRNA & Sheep & Iran & $\begin{array}{l}\text { (Farhang-Pajuh et al. } \\
\text { 2014) }\end{array}$ \\
\hline S. cruzi & 18S rDNA & Cattle & Malaysian & (Ng et al. 2015) \\
\hline S. capracanis & 18S rRNA & Goats & Malaysia & (Kutty et al. 2015) \\
\hline S. tenella & 18S rRNA & Sheep & Italy & (Bacci et al. 2016) \\
\hline S. arieticanis \& S. capracanis & 18S rDNA & Sheep & Brazil & (Bittencourt et al. 2016) \\
\hline S. tenella & 18S rDNA & Goats & Korea & (Hong et al. 2016) \\
\hline S. tenella & 18S rRNA & Sheep & Iraq & (Whaeeb and Faraj 2016) \\
\hline S. gigantea \& $S$. moulei & 18S rRNA & Sheep & Iran & (Kalantari et al. 2016) \\
\hline S. gigantea & 18S rRNA & Sheep & Argentina & (Gual et al. 2017) \\
\hline S. fusiformis \& S. moulei & 18S rRNA & Water buffaloes & Iraq & (Dakhil et al. 2017) \\
\hline S. tenella $\mathcal{E} S$. arieticanis & $\begin{array}{l}\text { 18S r RNA gene, } 28 \mathrm{~S} \text { r RNA, } \\
\text { coxı, and ITS-1 region }\end{array}$ & Sheep & China & (Hu et al. 2017) \\
\hline S. tenella $\mathcal{E}$ S. capracanis & 18S rRNA & Sheep \&goats & Tunisia & (Amairia et al. 2018) \\
\hline S. tenella & 18S rRNA & Sheep & Egypt & (Hussein et al. 2018) \\
\hline S. tenella and $S$. arieticanis & $\operatorname{cox} 1$ & Sheep & China & (Dong et al. 2018) \\
\hline S. giganteaE S. tenella & 18S rRNA & Sheep & Egypt & (Elmishmishy et al. 2018) \\
\hline S. fusiformis & 18S rRNA\&coxı & Buffaloes & China & (Mei Ren et al. 2019) \\
\hline S. cruzi $\mathcal{E} S$. hjorti & 18S rRNA & Cattle & Egypt & (El-kady et al. 2018) \\
\hline S. cruzi & 18S rRNA & Cattle & Korea & (Choi et al. 2018) \\
\hline S. tenella $\mathcal{E}$ S. capracanis & Coxi & Sheep \& goats & Saudi Arabia & (Metwally et al. 2019) \\
\hline S. bovifelis & $\begin{array}{l}\text { Cytochrome C Oxidase } \\
\text { subunit I mitochondrial } \\
\text { (mtDNA COI) gene }\end{array}$ & Cattle & Italy & (Rubiola et al. 2019) \\
\hline S. tenella and S. arieticanis. & $\begin{array}{l}\text { 18S rRNA, } 28 \mathrm{~S} \text { rRNA, coxı and } \\
\text { ITS-1 }\end{array}$ & Sheep & Egypt & (El-Morsey et al.2019) \\
\hline $\begin{array}{l}\text { S. arieticanis, } S \text {. tenella, S. gigantea, } \\
\text { S. medusiformis, ES. mihoensis }\end{array}$ & Cox1,18S and 28S rRNA & Sheep & Spain & (Gjerde et al. 2020) \\
\hline S. arieticanis & 18S rRNA & Sheep & Egypt & (Hussein et al.2020) \\
\hline Sarcocystis spp. & 18SrRNA & Sheep & Iraq & (Al-Saadi et al. 2020) \\
\hline S. gigantea, S. moulei, ES S. medusiformis & 18S rRNA & Sheep \& goats & Iraq & (Swar and Shnawa, 2020) \\
\hline
\end{tabular}

More recent genetic findings propose that $S$. medusiformis, S. gigantea and S. moulei have identical sister genome sequences. These species are documented to form macrosrcocysts in sheep, while cats serve asthe final host for them (Gjerde et al. 2020). This fact has also been detected in the cattle and water buffalo with uncommon Sarcocystis spp infection., Results of several other studies also suggest that Sarcocystis spp. are nonspecific for the intermediate host (Jehle et al. 2009; Xiang et al. 2011; Gjerde et al. 2016; Dakhil et al. 2017; El-kady et al. 2018).

Gjerde (2013a; 2013b) proved that the sequences of COX 1 are better than the ssRNA gene for identifying the closely associated spp of Sarcocystis and recommended it as a 
novel bio- genetic marker for further studies. Gjerde et al. (2016) recommended $\mathrm{COX}_{1}$ as a superior gene to $18 \mathrm{~S}$ and 28S rRNA genes as a bio-genetic marker. In the same aspect, El-Morsey et al. (2019) proved the priority of COX1 and ITS-1 genes as the best genetic markers compared to other genes to differentiate the closely related Sarcocystis spp owing to the high divergence among them. Another study evaluated the sequences of 4 genetic markers (18S rRNA, 28S rRNA, mitochondrial COX1 and ITS-1) specific for $S$. tenella and $S$. arieticanis.

It has been confirmed that the ITS-1 region is more helpful for distinguishing the closely related Sarcocystis spp. because of their high divergence (Hu et al. 2017). The same was true for the genetic resemblance between sheep and goats infection with Eimeria spp., which was phylogenetically confirmed by the ITS-1 sequences technique in Egypt. The sequence of ITS-1 gene of $E$. ahsata was $100 \%$ identical with E. ahsata and clustered in one clade with E. cardinalis and E. faurei. Alternatively, the similarity was $100 \%$ between E. arloingi and E. arloingi of goats. It is clustered with E. ellipsoidalis of bovine sources (Hassanen et al. 2020). Conventionally, the Sarcocystis' ultrastructural features are considered as fundamentals for the identification of numerous Sarcocystis spp. within the same intermediate host ( $\mathrm{Hu}$ et al. 2017; Huang et al. 2019). Currently, genomic sequence analysis is an essential technique to clarify whether morphologically similar Sarcocystis spp. from different intermediate hosts are the same species or not. Findings of several recent studies concerning the molecular characterization of Sarcocystis spp isolated from meatproducing animals have been summarized in Table 2.

\section{Conclusion}

Recently, researchers achieved outstanding success in molecular aspects to identify various Sarcocystis spp that infect livestock. Various molecular techniques evaluated for this purpose are 18S rRNA, 28S rRNA, 18S rDNA, mitochondrial cytochrome $\mathrm{C}$ oxidase subunit 1 gene (COX 1$)$, and ITS-1 region. Usually, the Sarcocystis' ultrastructural features are considered as fundamentals for the identification of many Sarcocystis spp. within the same intermediate host. At present, genomic sequence analysis is necessary to clarify whether morphologically similar Sarcocystis from different intermediate hosts are the same species or not. Sarcocystis spp. are known as primarily host-specific, but a large number of Sarcocystis spp using different animals as intermediate hosts were identified during the last few years. As a result, the issue of host specificity of Sarcocystis species remains questionable.

\section{REFERENCES}

Abdel-Baki AA et al., 2009. Lambs infected with UVattenuated sporocysts of Sarcocystis ovicanis produced abnormal sarcocysts and induced protective immunity against challenge infection. Korean Journal of Parasitology 47: 131-138.
Abdel-Ghaffar F et al., 2009. Life cycle of Sarcocystis camelicanis infecting the camel (Camelus dromedarius) and the dog (Canis familiaris), light and electron microscopic study. Parasitology Research 106: 189-195.

Al-Hoot AS et al., 2005. Microscopic study on Sarcocystis moulei from sheep and goats in Saudi Arabia. Journal of the Egyptian Society of Parasitology 35: 295-312.

Al-Quraishy S et al., 2014. Sarcocystis arieticanis (Apicomplexa: Sarcocystidae) infecting the heart muscles of the domestic sheep, Ovis aris (Artiodactyla: Bovidae), from K. S. A. on the basis of light and electron microscopic data. Parasitology Research 113: 3823-3831.

Al-Saadi SA et al., 2020. Molecular identification of Sarcocystis species infection in sheep in Karbala Governorate-Iraq. Medico Legal Update 20: 889-895.

Amairia S et al., 2018. First detection and molecular identification of Sarcocystis spp. in small ruminants in North-West Tunisia. Transboundary and Emerging Diseases, 65(2), 441-446.

Bacci $C$ et al., 2016. Detection of Toxoplasma gondii and Sarcocystis tenella in indigenous CornigLiese sheep in Italy using serological and molecular methods. Small Ruminant Research 135: 13-16.

Bahari P et al., 2014. Molecular identification of macroscopic and microscopic cysts of sarcocystis in sheep in North Khorasan province, Iran. International Journal of Molecular and Cellular Medicine 3: 51-56.

Bittencourt MV et al., 2016. Sarcocystis spp. in sheep and goats: frequency of infection and species identification by morphological, ultrastructural, and molecular tests in Bahia, Brazil. Parasitology Research 115: 1683-1689

Blazejewski T et al., 2015. Systems-based analysis of the Sarcocystis neurona genome identifies pathways that contribute to a heteroxenous life cycle. MBio 6: 1-16.

Choi TI et al., 2018. Detection and identification of Sarcocystis cruzi (Protozoa: Apicomplexa) by molecular and ultrastructural studies in naturally infected Korean cattle (Bos taurus coreanae) from Daejeon, Korea. The Korean Journal of Parasitology 56: 121-127.

Collins GH and Charleston WAG, 1979. Studies on Sarcocystis species IV: A species infecting dogs and goats; development in goats. New Zealand Veterinary Journal 27: 260-262.

Collins GH et al., 1979. Studies on Sarcocystis species. III: the macrocystic species of sheep. New Zealand Veterinary Journal 27: 204-206.

Dakhil HG et al., 2017. Molecular identification of Sarcocystis fusiformis and S. moulei infecting water buffaloes (Bubalus bubalis) in southern Iraq. World Journal of Pharmaceutical Research 6: 215-229.

Da Silva RC et al., 2009. First identification of Sarcocystis tenella (Railliet, 1886) Moulé, 1886 (Protozoa: Apicomplexa) by PCR in naturally infected sheep from Brazil. Veterinory Parasitology 165: 332-336.

Dong $\mathrm{H}$ et al., 2018. Sarcocystis species in wild and domestic sheep (Ovis ammon and Ovis aries) from China. BMC Veterinary Research 14: 377. 
Dubey JP et al., 1981. Sarcocystosis in goats: clinical signs and pathologic and hematologic findings. Journal of the American Veterinary Medical Association 178: 683-699.

Dubey JP et al., 1988. Sarcocystis arieticanis and other Sarcocystis species in sheep in the United States. Journal of Parasitology 74: 1033-1038.

Dubey JP et al., 1989. Sarcocystosis of animals and man. CRC Press, Boca Raton, Florida.

Dubey JP et al., 1991. Sarcocystis neurona n. sp. (Protozoa: Apicomplexa), the etiologic agent of equine protozoal myeloencephalitis. Jouranal of Parasitology 77, 212- 218.

Dubey JP et al., 2014. Sarcocystis cafferin. sp. (Protozoa: Apicomplexa) from the African buffalo (Syncerus caffer). Journal of Parasitology 100: 817-827.

Dubey JP et al., 2015. Sarcocystis heydorni n. sp. (Apicomplexa: Sarcocystidae) with cattle (Bos taurus) and human (Homo sapiens) cycle. Parasitology Research 114: 4143-4147.

Dubey JP and Lindsay DS, 2006. Neosporosis, toxoplasmosis, and sarcocystosis in ruminants. Veterinary Clinics of North America: Food Animal Practice 22: 645-671.

Dubey JP, 2015. Foodborne and waterborne zoonotic sarcocystosis. Food and Waterborne Parasitology 1: 211.

Dubey JP et al., 2016. Sarcocystosis of animals and humans. 2nd Edn. Boca Raton: CRC Press; Taylor \& Francis Group,

Dubey JP et al., 2014. Sarcocystis cafferin. sp. (Protozoa: Apicomplexa) from the African buffalo (Syncerus caffer). Journal of Parasitology 100: 817-827.

El-kady AM et al., 2018. First molecular characterization of Sarcocystis spp. in cattle in Qena Governorate, Upper Egypt. Journal of Parasitic Diseases 42: 114-121.

Elmishmishy B et al., 2018. Genetic variability within isolates of Sarcocystis species infecting sheep from Egypt. Veterinary Parasitology: Regional Studies and Reports 13: 193-197.

El-Morsey A et al., 2019. Ultrastructural and molecular identification of the sarcocysts of Sarcocystis tenella and Sarcocystis arieticanis infecting domestic sheep (Ovis aries) from Egypt. Acta Parasitologica 64: 501-513.

Farhang-Pajuh F et al., 2014. Molecular determination of abundance of infection with Sarcocystis species in slaughtered sheep of Urmia, Iran. Veterinary Research Forum 5: 181-186.

Fayer R, 1972. Gametogony of Sarcocystis sp. in cell culture. Science 175: 65-67.

Fayer R, 2004. Sarcocystis spp. in human infections. Clinical Microbiology Review 17: 894-902.

Fayer R and Johnson AJ. 1973. Development of Sarcocystis fusiformis in calves infected with sporocysts from dogs. Journal of Parasitology 59: 1135-1137.

Fayer R and Johnson A, 1975. Effect of amprolium on acute sarcocystosis in experimentally infected calves. Journal of Parasitology 61: 932-936.

Fayer R et al., 1976. Abortion and other signs of disease in cows experimentally infected with Sarcocystis fusiformis from dogs. Journal of Infectious Diseases 134: 624--628.

Fayer R et al., 2015. Human infections with Sarcocystis species. Clinical Microbiology Reviews 28: 295-311.

Formisano $\mathrm{P}$ et al., 2013. Identification of Sarcocystis capracanis in cerebrospinal fluid from sheep with neurological disease. Veterinary Parasitology 193: 252-255.

Ghaffar FA et al., 1989. The fine structure of cysts of Sarcocystis moulei from goats. Parasitology Research, 75: 416-418.

Gjerde B, 2013a. Phylogenetic relationships among Sarcocystis species in cervids, cattle and sheep inferred from the mitochondrial cytochrome c oxidase subunit I gene. International Journal of Parasitology 43: 579-591.

Gjerde B, 2013b. Sarcocystis species in red deer revisited: with a re-description of two known species as Sarcocystis elongatan. sp. and Sarcocystis truncata n. sp. based on mitochondrial sequences. Parasitology 141: 441-452.

Gjerde B et al., 2016. Molecular differentiation of Sarcocystis buffalonis and Sarcocystis levinei in water buffaloes (Bubalus bubalis) from Sarcocystis hirsuta and Sarcocystis cruzi in cattle (Bos taurus). Parasitology Research 115: 2459-2471.

Gjerde B et al., 2020. Molecular characterization of five Sarcocystis species in domestic sheep (Ovis aries) from Spain. Parasitology Research 119: 215-231.

Gual 1 et al., 2017. Molecular confirmation of Sarcocystis gigantea in a naturally infected sheep in Argentina: A case report. Veterinary Parasitology 248: 25-27.

Hassanen EAA et al., 2020. Prevalence and phylogenetic analysis of Eimeria species in sheep and goats in Sharkia Governorate, Egypt. Pakistan Veterinary Journal. 40: 437-442.

Hong EJ et al., 2016. Ultrastructural and molecular identification of Sarcocystis tenella (Protozoa, Apicomplexa) in naturally infected Korean native goats. Veterinární Medicína 61: 374-381.

$\mathrm{Hu} \mathrm{JJ}$ et al., 2017. Sarcocystis spp. in domestic sheep in Kunming City, China: prevalence, morphology, and molecular characteristics. Parasitology 24: 30.

Huang $\mathrm{Z}$ et al., 2019. Morphological and molecular characterizations of Sarcocystis miescheriana and Sarcocystis suihominis in domestic pigs (Sus scrofa) in China. Parasitology Research 118: 3491-3496.

Hussein NM et al., 2018. Morphological, ultrastructural, and molecular characterization of Sarcocystis tenella from sheep in Qena governorate, upper Egypt. Egyptian Academic Journal of Biological Sciences 10: 11-19.

Hussein NM, 2020. Morphological and molecular characterization of Sarcocystis arieticanis from the heart muscles of domestic sheep, Ovis aries, in Qena, upper Egypt. Journal Advanced Veterinary Research 10: $73-80$.

Heydorn AO et al., 1975. Proposal for a new nomenclature of the Sarcosporidia. Zeitschrift für Parasitenkunde 48: 73-82. 
Jehle C et al., 2009. Diagnosis of Sarcocystis spp. in cattle (Bos taurus) and water buffalo (Bubalus bubalis) in Northern Vietnam. Veterinary Parasitology 166: 314320.

Kalantari N et al., 2016. Molecular analysis of Sarcocystis spp. isolated from sheep (Ovis aries) in Babol area, Mazandaran Province, Northern Iran. Iran Journal of Parasitology 11: 73-80.

Kutty MK et al., 2015. Detection of sarcocystosis in goats in Malaysia by light microscopy, histology, and PCR. Tropical Animal Health Production 47: 751-756.

Lankester ER, 1882. On Drepanidium ranarum, the cellparasite of the frog's blood and spleen (Gaule's Würmschen). QJ Microscience Science 22:53-65.

Leger L, 1911. Caryospora simplex, coccidie monosporee et la classification des coccidies. Archiv fur Protistenkunde 22: 71-86.

Leger L and Duboscq O, 1910. Selenococcidium intermedium $\mathrm{L}$. et $\mathrm{D}$. et la systematique des sporozoaires. Archives de Zoologie experimentale et generale 5: 187-238.

Levine ND, 1970. Taxonomy of the Sporozoa. Journal of Parasitology 56(II): 208-209.

Leuckart R, 1879. Die Parasiten des Menschen, 2nd ed. G. F. Winter, Leipzig, Germany, viii $+336 \mathrm{p}$

Levine ND, 1986. The taxonomy of Sarcocystis (Protozoa, Apicomplexa) species. Journal Parasitology 72: 372.

Lindsay DS and Dubey JP, 2020. Neosporosis, Toxoplasmosis, and Sarcocystosis in ruminants. The Veterinary Clinics of North America. Food Animal Practice 36: 205-222.

Mandour AM, 1969. Sarcocystis nesbitti n. sp. from the rhesus monkey. The Journal of Protozoology 16: 353354.

Markus MB et al., 2004. Sarcocystosis. Chapter 20 in: Coetzer, JAW and Tustin, RC (eds). Infectious Diseases of Livestock. Cape Town, Oxford University Press Pp. 360-375.

Mehlhorn H, 2016. Sarcocystis species: Animals as intermediate hosts. In: Mehlhorn H. (eds) Encyclopedia of Parasitology. Springer, Berlin, Heidelberg.

Mei REN et al., 2019. Molecular characterization of Sarcocystis species isolated from Chinese buffaloes in Guizhou province based on 18S rRNA and cox1 sequences, Mitochondrial DNA Part B, 4: 637-641.

Metwally DM et al., 2019. Molecular characterization of Sarcocystis species isolated from sheep and goats in Riyadh, Saudi Arabia. Animals 9: 256.

Miescher F, 1843. Über eigenthümliche Schläuche in den Muskeln einer Hausmaus. Bericht der Verhandlungen der Naturforschenden Gesellschaft 5: 198 - 202.

$\mathrm{Ng} \mathrm{YH}$ et al., 2015. Genetic variants of Sarcocystis cruzi in infected Malaysian cattle based on 18S rDNA. Research in Veterinary Science 103: 201-204.

Odening K, 1998. The present state of species-systematics in Sarcocystis Lankester, 1882 (Protista, Sporozoa, Coccidia). Systematic Parasitology 41: 209-233.

Poche F, 1913. Das System der Protozoa. Archiv fur
Protistenkunde 30: 125-321.

Rommel M et al., 1972. Contribution on the life cycle of Sarcocporidia. I. the sporocyst of Sarcocystis tenella in the feces of the cat. Berl Munich Tierztl Wochenscher. 85: 101-105.

Rubiola S et al., 2019. Molecular identification of Sarcocystis spp. in cattle: partial sequencing of Cytochrome C Oxidase subunit 1 (COI). Italian Journal of Food Safety 7: 7725.

Ruiz A and Frenkel JK, 1076. Recognition of cyclic transmission of Sarcocystis muris by cats. Journal of Infectious Diseases 133: 409-418.

Shahbazi A et al., 2013. Identification of Sarcocystis tenella and Sarcocystis arieticanis isolated from slaughtered sheep in Tabriz abattoir using parasitological and PCR-RFLP methods. Journal of Comparative Pathobiology Iran 10: 959-964.

Swar SO, 2021. Ultrastructural and molecular characterization of Sarcocystis species in domestic sheep and goats from Soran city, Erbil-Iraq. PhD Thesis. Department of Biology, Faculty of Science, Soran University, Iraq.

Swar SO and Shnawa BH, 2020. Ultrastructural and molecular characterization of Sarcocystis species derived from macroscopic Sarcocysts of domestic sheep and goats in Soran city, Erbil, Iraq. World Veterinary Journal 10: 540-550.

Swar SO and Shnawa BH, 2021. Recent advances in molecular characterization of Sarcocystis species in some meat producing animals: An updated review. Asian Journal of Agriculture and Biology 2021: 1-10.

Tappe D et al., 2013. Initial patient cluster and first positive biopsy findings in an outbreak of acute muscular Sarcocystis-like infection in travellers returning from Tioman island, Peninsular Malaysia, in 2011. Journal of Clinical Microbiology 51: 725-726.

Tenter AM et al., 1992. Phylogenetic relationships of Sarcocystis species from sheep, goats, cattle and mice based on ribosomal RNA sequences. International Journal for Parasitology 22: 503-513.

Underwood WJ et al., 2015. Biology and Diseases of Ruminants (Sheep, Goats, and Cattle). Laboratory Animal Medicine. Academic Press 623-694.

Wallace GD, 1973. Sarcocystis in mice inoculated with Toxoplasma-like oocysts from cat feces. Science 180: 1375-1377.

Whaeeb ST and Faraj AA, 2016. Molecular identification and phylogeny of microscopic Sarcocystis sheep in Baghdad Province. International Journal of Advanced Research in Biological Sciences 3: 50-56.

Yang $\mathrm{Z}$ et al., 2001. Analysis of the 18S rRNA genes of Sarcocystis species suggests that the morphologically similar organisms from cattle and water buffalo should be considered the same species. Molecular and Biochemical Parasitology 115: 283-288.

Xiang Z et al., 2011. Sarcocystis cruzi: Comparative studies confirm natural infections of buffaloes. Experimental Parasitology 127: 460-466. 